\title{
Pharmaceutical care in transplantation: current challenges and future opportunities
}

\author{
Jordan R Covvey*,‡,1 \& Erin E Mancl ${ }^{\ddagger} 2$ \\ ${ }^{1}$ Assistant Professor in Pharmacy Administration, Division of Pharmaceutical, Administrative \& Social Sciences, Duquesne University \\ School of Pharmacy, 600 Forbes Ave, 418 Mellon Hall, PA 15282, USA \\ ${ }^{2}$ Medical Science Liaison, Mallinckrodt Pharmaceuticals, Somerset Center, 1425 US Route 206, NJ 07921, USA \\ *Author for correspondence: Tel.: +1 412396 2636; covveyj@duq.edu \\ $\ddagger$ Authors contributed equally

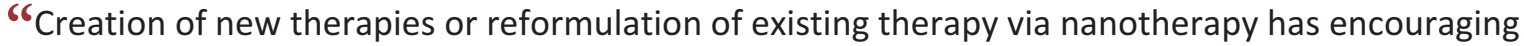 potential to increasing opportunity for localized drug delivery, expand drug solubility and minimize toxicities"

First draft submitted: 11 March 2019; Accepted for publication: 9 August 2019; Published online: 15 October 2019

Keywords: antimetabolites $\bullet$ calcineurin inhibitors $\bullet$ immunosuppression $\bullet$ nanoparticle $\bullet$ sirolimus $\bullet$ transplantation

The United Network for Organ Sharing, the nonprofit organization that contracts with the government to manage the country's organ transplantation system, reports that over 750,000 transplants have been performed in the USA since 1988 [1]. Over 113,000 individuals are currently listed as candidates on waiting lists for organs [1]. In 2016, just over 23,000 referrals to organ procurement organizations met criteria for eligible or imminent death per the Organ Procurement and Transplantation Network definition, leading to almost 10,000 deceased donors for transplant [2].

Although a variety of clinical factors among the donors and recipients affect the success of individual solid organ transplants, outcomes and developments across the field continue to broadly improve over time. Kidney, liver and heart transplants collectively account for approximately $90 \%$ of procedures [1]. For kidney transplantation, all-cause graft failure at 6 months for deceased donor recipients declined 36\% from 2005 to 2015 [3]. For living donor kidneys, nearly $82 \%$ of those transplanted in 2006 were still functioning 10 years post-transplant (censored for death) [3]. Over the past decade, graft survival has steadily improved for liver recipients, with 1-year failure rates in 2015 estimated at 10.5 and 5.1\% for deceased and living donor transplants, respectively [4]. 1- and 5-year survival for heart transplants in 2009-2011 was 90.1 and 78.3\%, with overall rates of death post-transplant decreased since 2005 [5]. Most recently, the field has seen expansion and preliminary successes in vascularized composite allotransplantation (VCA), or the transplantation of multiple tissues as a functional unit [6,7].

Despite considerable progress since the advent of transplantation in the 1950s, there does remain room for improvement. For instance, long-term patient survival for lung transplantation remains low and unchanged over time, estimated at $55.6 \%$ at 5 years [8]. Wide geographic variability is seen among liver transplantation, with significant differences in the average Model for End-Stage Liver Disease score at the time of transplant that has caused a fair degree of controversy across the literature [4,9]. Disparities are evident within kidney transplantation, where living donor transplants occur in $65.1 \%$ of white recipients, but only $12.3 \%$ of black recipients, despite almost equal racial distribution on the waiting list [3]. Furthermore, more than $10 \%$ of individuals on the waiting list for kidney transplants are for repeat procedures [10], signaling opportunities for improvement in the prevention of acute and chronic rejection.

Advances are particularly needed in the immunosuppressive regimens that are essential in the peri- and posttransplant periods across all organs [11]. As lifelong therapies, these medicines are essential in the prevention of rejection and long-term graft success, yet considerably increase the risk for infection and other toxicities. This article will review the major pharmacotherapy strategies used in solid organ transplant, along with some of the difficulties in using these therapies, and a look forward toward new opportunities and development. 


\section{The landscape of immunosuppression}

Complex pharmacotherapy regimens for immunosuppression remain necessary for graft survival in the solid organ transplant recipient. While treatment may simplify if time, graft function and rejection potential permit, many patients remain on lifelong dual, triple or quadruple immunosuppression. In one cross-sectional analysis using the Medication Regimen Complexity Index, scores for kidney (17.9 \pm 8.1) and liver (18.1 \pm 8.8$)$ transplant recipients were significantly higher for other chronic diseases, which broadly ranged from 3 to 7 on the Medication Regimen Complexity Index [12]. These therapies are additionally accompanied by narrow therapeutic indices, significant interpatient variability and high potential for drug interactions through both pharmacokinetic and pharmacodynamic processes. The major metabolic/transport pathways for immunosuppressant agents (e.g., CYP450 isoenzyme 3A4 and 3A5, UDP-GT and P-gp) play important roles for a host of other medications, heightening risk through induction, inhibition and substrate interactions. Most recipients also have pre-existing co-morbid conditions beyond their transplant needs (each often requiring their own pharmacotherapy), and further complications can arise with iatrogenic co-morbidities emerging from the use of immunosuppressive therapy itself, such as hypertension and diabetes mellitus. Each immunosuppressive class or agent targets unique molecular receptors to suppress lymphocyte activation and proliferation. In addition to temporary or lifelong corticosteroids, major options for maintenance immunosuppression include calcineurin inhibitors (CNI), antimetabolites and mammalian (mechanistic) target of rapamycin (mTOR) inhibitors.

\section{Calcineurin inhibitors}

After the discovery and clinical use of key CNI compounds cyclosporine and tacrolimus, graft survival in solid organ transplant increased significantly [13,14]. Despite their narrow therapeutic index, they remain a cornerstone of solid organ transplant pharmacotherapy through inhibition of T-lymphocyte activation and impairment of lymphocyte function and replication [15]. Frequent (i.e., weekly or even twice weekly) laboratory monitoring is often required to adjust total daily dosing to achieve goal trough concentrations, since elevated trough concentrations are associated with significant toxicities, while area under the curve concentrations below target are associated with acute and chronic cellular rejection [16].

Despite their efficacy successes, the adverse effects associated with CNIs can be substantial, including abnormal hair growth (e.g., alopecia with tacrolimus and hirsutism with cyclosporine), electrolyte and metabolic abnormalities (e.g., glucose intolerance, hyperlipidemia, hyperkalemia), gastrointestinal disturbances (e.g., anorexia, nausea, vomiting), hypertension, neurotoxicities (e.g., tremor, headache, visual abnormalities) and nephrotoxicity [17]. Due to their major absorption and metabolism through P-gp and CYP450 isoenzymes 3A4 and 3A5, significant dosing variability is required when considering individual patient's enzyme phenotype as well as numerous drug-drug interactions that affect the function of P-gp and CYP enzyme system. In fact, it has been demonstrated that CYP3A5 polymorphisms associated with normal or intermediate function are associated with higher total daily doses, which may increase risk of chronic CNI toxicities [18-20].

Pharmacogenomic tools that help predict metabolism and serum concentrations are often used in addition to clinical expertise to initiate and maintain CNI dosing. In fact, some centers require CYP3A5 genotype testing be completed during work-up for transplant listing, particularly in at-risk populations (e.g., African-Americans). If a normal or intermediate function CYP3A5 polymorphism is identified, clinicians may decide to employ one of more of the following strategies: more aggressive CNI dosing; purposeful addition of CYP3A4 and 3A5 isoenzyme inhibitors to decrease CNI metabolism; or utilization of more aggressive induction strategies in the absence of contraindications. Despite the known limitations with CNI dosing, it is unclear that pharmacogenomic testing actually improves outcomes. In a 2010 study, 280 patients receiving kidney transplants were prospectively assigned to receive tacrolimus using a standard regimen, or one adapted based on patient-specific CYP3A5 genotype [21]. Patients in the latter group receiving pharmacogenetic-informed dosing were more likely to achieve target drug concentrations 3 days post-initiation ( $43.2 \mathrm{vs} 29.1 \%$; $\mathrm{p}=0.03$ ), were able to reach their targets more rapidly and were less likely to require dose changes, compared with standard concentration-informed dosing [21]. However, no changes in clinical end points (e.g., graft survival, acute rejection, delayed graft function, drug toxicity) were seen in this study [21], nor in a longer-term follow-up of the patients [22]. A second randomized controlled trial published in 2016 evaluating a similar comparison among 240 kidney transplant patients failed to identify a difference in target concentration achievement on day $3(37.4$ vs $35.6 \% ; \mathrm{p}=0.079$ for standard and adapted dosing, respectively) or across any clinical outcomes [23]. Accordingly, it is likely that the pharmacokinetics of 
tacrolimus are influenced importantly by polymorphisms beyond CYP3A5, and that transplant outcomes are similarly varied in their influences.

From a formulation perspective, more recently, the US FDA approved a novel extended-release formulation of tacrolimus (Envarsus XR ${ }^{\circledR}$ ), which includes a proprietary technology (MeltDose) that frames tacrolimus particles into a 'solid solution', permitting a slower, more consistent absorption throughout the entire small bowel [24,25]. Since earlier achievement of therapeutic levels of a CNI provides greater protection against acute rejection [26], the technology is particularly hopeful. However, similar rates of toxicities still exist compared with conventional tacrolimus and access for all solid organ transplant patients is limited, since the product is only FDA-approved in kidney recipients $[27,28]$.

\section{Antimetabolites}

Antimetabolites are also frequently used in solid organ transplant, including the two available agents azathioprine and mycophenolate mofetil (MMF), which both lead to impairment in T- and B-lymphocytes. Azathioprine is metabolized to 6-mercaptopurine and eventually to 6-ionosine monophosphate, which inhibits purine synthesis [29]. MMF is converted to mycophenolic acid, a noncompetitive inhibitor of IMPDH [29].

MMF is often preferred over azathioprine in kidney transplantation due to a preferable adverse effect profile and reduced risk of graft rejection [30], as well as improved graft survival in lung and heart recipients [31]. However, some considerations in its use are necessary. MMF is metabolized by CYP3A4/5 and UDP-GT pathways, increasing the potential for drug-drug interactions. It is contraindicated among women of child-bearing age due to teratogenicity [32], and associated with dose-related hematological effects, particularly leukopenia and gastrointestinal effects (e.g., diarrhea, nausea and vomiting). Recent animal data suggest that MMF-associated diarrhea may be spared in recipients who do not have an intact gut microbiome [33]. Whether strategies to alter a patient's microbiome can improve tolerance to MMF are yet to be evaluated. Therefore, patients with intolerable gastrointestinal side effects are often converted to azathioprine with some success. Hematological toxicities are also associated with azathioprine, and are more likely to occur in patients with deficiency in TPMT and/or NUDT15 activity. Therefore, clinicians often perform genotyping in patients to determine risk and/or cause of adverse effects. Finally, because azathioprine is converted to its metabolite via xanthine oxidase, inhibitors such as allopurinol can result in significant toxicities.

\section{mTOR inhibitors}

mTOR inhibitors bind to the FK-binding protein to inhibit the activity of mTOR and block the response of T- and B-lymphocytes to cytokines [34]. Their original development was aimed as a replacement or minimization strategy for CNIs (due to associated nephrotoxicity), but this was never fully realized due to less than optimal performance in comparative clinical trials [35,36]. Nevertheless, mTOR inhibitors are frequently used for renal sparing effects in organ transplant recipients. In addition, mTOR inhibitors are associated with low incidence and possible slowing of chronic rejection in thoracic transplant recipients [37]. Today, although not used in de novo solid organ transplant, mTOR inhibitors sirolimus (rapamycin) and everolimus may be utilized in later stages of maintenance immunosuppression in place of a component, or in augmentation with a patient's initial regimen [38].

Like CNIs, mTOR inhibitors possess a narrow therapeutic index, and must have trough concentrations monitored during initiation, after dose adjustment or after drug-drug interaction changes. In addition, due to their complex mechanism, there is an urgent need for efficient biomarkers to predict efficacy of mTOR-based therapy and avoid toxicity in patients who will indeed not benefit. Adverse effects with the class are not insignificant, and include hematological effects, metabolic and gastrointestinal abnormalities like the CNIs, impaired wound healing and interstitial pneumonitis, among others [39].

Sirolimus interestingly has been associated with increased mortality (hazard ratio: 1.43; 95\% CI: 1.21-1.71) after kidney transplant in a meta-analysis of 21 randomized controlled trials, primarily associated with cardiovascular and infective complications [40]. This effect was seen in both de novo and conversion uses of the drug [40]. Similar increased risk of fatal adverse events (relative risk: 3.24; 95\% CI: 1.21-8.67) has been noted in a different metaanalysis of 12 trials among patients with malignancy taking everolimus or temsirolimus (an mTOR inhibitor not utilized for transplant) [41], suggesting that this may be a class effect. The exact cause and significance of this effect is unknown, particularly as it has not been associated with allograft loss [42], but it adds further difficulty to the utility of mTOR inhibitors in solid organ transplantation. 


\section{Economic \& humanistic impacts}

Barriers in the provision of effective immunosuppression are not confined to the clinical aspects of these therapies. Financial concerns remain paramount for patients undergoing transplantation. Estimated billed charges per transplant in 2017 ranged from $\$ 347,000$ for pancreas, $\$ 414,800$ for kidney and as high as $\$ 1.38$ million for heart transplantation [43]. Although hospital admissions for the procedures themselves compose the bulk of the cost, outpatient medications for immunosuppressants and other necessary therapies are also significant, generally reaching over $\$ 20,000$ per patient in the first 180 days after discharge [43]. The Medicare program covers transplant procedures under Part A and B for patients with end-stage renal disease receiving dialysis, but outpatient coverage for immunosuppressive therapies post-transplant generally ends 36-month post-transplant if the beneficiary is not Medicare-eligible as a function of age or disability. Instances like this cause considerable difficulty in maintaining graft success long-term, particularly for recipients without significant financial resources. In a 2010 survey of all kidney transplant programs across the USA, more than $70 \%$ of programs reported problems with patients affording their medications, and 68\% could directly attribute patient deaths and graft loss as a result of medication nonadherence associated with affordability [44].

The humanistic impacts of currently available immunosuppressive therapies are also relevant, particularly for patients who experience adverse effects. A prospective multicenter study before and up to 2 years post-liver transplantation suggested that persistent anxiety and depression were common (23 and 29\%, respectively) [45]. Furthermore, these symptoms were associated with adverse effects stemming from immunosuppressive therapy, as well as decreases in adherence and health-related quality of life (QoL) [45]. Among adults more than 12 months post-kidney transplant, nonadherence with immunosuppressive therapy (seen in 33.6\% of patients) was associated with lower mental health-related QoL and lower perceived social support [46]. And yet, despite knowing the importance of measures such as QoL, a recent systematic review of randomized controlled trials of maintenance immunosuppression after kidney transplantation found infrequent inclusion of patient-reported measures of well being $[47]$.

\section{Encouraging developments in immunosuppression}

One area that may provide promising potential to overcome the barriers of traditional immunosuppression is nanotherapy. Creation of new therapies or reformulation of existing therapy via nanotherapy has encouraging potential to increasing opportunity for localized drug delivery, expand drug solubility and minimize toxicities $[48,49]$. There is also opportunity in nanoparticle-based delivery to be useful in a larger transplant arena, utilized as pretreatment of organs prior to transplantation for endothelial cell protection, incorporated in strategies to expand donor organs through use of allografts previously thought to be unsuitable, or in efforts to protect from ischemia-reperfusion injury [50]. Preliminary scientific work in the advancement of immunosuppression post-transplant has shown encouraging results. One example is the development and initial results of a rapamycin nanoimmunotherapy in a mouse model for heart transplantation, which demonstrated high encapsulation efficacy and cellular specificity [51,52]. Use of this strategy (in concert with a second nanotherapy augmenter) resulted in allograft survival rates greater than $70 \%$ at 100 days post-transplant, compared with $100 \%$ rejection before day 10 among mice receiving placebo [52].

Other nanoparticle delivery strategies are also being explored to optimize existing therapies. Nanoparticle delivery formulations of CNI, antimetabolite or mTOR inhibitors are in their infancy, with limited evaluations in animal models as a means to overcome the current variability and toxicity with standard systemic therapy. In one example, researchers trialed a hydrogel formulation of tacrolimus with controlled release triggered alongside inflammatory proteolytic enzymes; in a rat hind-leg VCA model, median graft survival for the hydrogel-treated rat extended past 100 days, in comparison to 11 days for control groups [53]. Another study utilized a topical formulation of mycophenolic acid (overcoming the barriers of skin esterases on MMF) to assess permeation and pharmacokinetic characterization in rats and identified higher tissue and lower systemic drug levels that may be useful for future application in VCA [54]. In another animal model, inhaled nanoparticle tacrolimus demonstrated similar efficacy to systemic tacrolimus with significantly lower serum concentrations, a strategy that could minimize current toxicity rates if studied in humans [55]. Finally, a number of reports have demonstrated improvement in lung function with the off-label use of nebulized CNI in patients with chronic lung allograft dysfunction [56-60]. In fact, the most recent randomized placebo-controlled trial demonstrated a survival advantage with the addition of the nebulized cyclosporine [58]. The benefits of such therapy in chronic lung allograft dysfunction may be related to drug dispersion in areas of the lung which remain well ventilated. Despite these preliminary findings, an FDA-approved formulation 
of aerosolized cyclosporine or tacrolimus is not available. Further research is also needed to confirm whether there is a role for nebulized CNI beyond salvage therapy in lung recipients.

\section{Future directions for transplantation}

Despite the advent of modern immunosuppression, the morbidity associated with nonspecific lymphocyte inhibition is significant. Chronic diseases and infections, even with prophylaxis augmentation, are prevalent in solid organ transplant recipients. Current and future strategies to reduce exposure to immunosuppression include proactively identifying patients who may be more likely to experience rejection or who are acutely experiencing rejection.

Allomap ${ }^{\circledR}$ and Allosure ${ }^{\circledR}$ are noninvasive blood tests available for kidney and heart transplant recipients that can either detect genetic components of rejection or donor-derived cell-free DNA. With Allomap, heart transplant recipients can be risk-stratified for rejection using gene expression profiling [61-65]. With Allosure, kidney transplant recipients can be tested for elevations in fragments of donor-derived cell-free DNA, a biomarker signifying tissue injury [66-68] and acute rejection. There is growing interest in using these same assays in other solid organ transplant recipients, particularly lung transplant [69], which carries a high rate of acute and chronic rejection.

On the opposing spectrum, there is focus on detecting patients at the highest risk of opportunistic infections. Solid organ transplant recipients are at risk of cytomegalovirus (CMV) infection activation or reactivation while on maintenance immunosuppression. There is growing evidence that assays specific for CMV T-cells can be performed and may useful in part of a risk-stratification algorithm in pre-transplant candidates, or even predicting patients experiencing early CMV disease [70-72]. Furthermore, therapeutic adoptive T-cell therapy has been used as salvage, therapy for severe CMV disease [73] and is being studied for more routine clinical use in the era T-cell infusions for leukemias.

Ultimately, many are excited at the prospect of transplant methods that would significantly reduce or potentially avoid the need for immunosuppression altogether. Simultaneous stem cell and solid organ transplant is sometimes indicated, and often results in a reduced need for immunosuppression after transplant. In addition, donor-specific T-regulatory cell therapy is being evaluated for kidney transplant recipients [74] and could likely be used in other solid organ transplants. Regeneration of organs through stem cells is another area of fast-moving research [75], and may someday be possible.

\section{Conclusion}

Solid organ transplantation has seen significant successes over the past several decades through the use of highly effective immunosuppression. Despite availability of a variety of therapies, adverse effects from complex drug regimens and graft failure continue to be prevalent, alongside significant financial and humanistic impacts for patients receiving these therapies. The need for individualized therapy to optimize outcomes is clear, and encouraging scientific developments in the pipeline are focused on the optimization of existing formulations through strategies such as nanoimmunotherapy. With continued scientific advancement in the field of transplantation, there will be significant opportunity to improve the length and QoL for patients worldwide.

Financial \& competing interests disclosure

The authors have no relevant affiliations or financial involvement with any organization or entity with a financial interest in or financial conflict with the subject matter or materials discussed in the manuscript. This includes employment, consultancies, honoraria, stock ownership or options, expert testimony, grants or patents received or pending, or royalties.

No writing assistance was utilized in the production of this manuscript.

\section{References}

1. United Network for Organ Sharing. Data. https://unos.org/data/

2. Israni AK, Zaun D, Rosendale JD, Schaffhausen C, Snyder JJ, Kasiske BL. OPTN/SRTR 2016 annual data report: deceased organ donation. Am. J. Transplant. 18(Suppl. 1), 434-463 (2018).

3. Hart A, Smith JM, Skeans MA et al. OPTN/SRTR 2016 annual data report: kidney. Am. J. Transplant. 18(Suppl. 1), 18-113 (2018).

4. Kim WR, Lake JR, Smith JM et al. OPTN/SRTR 2016 annual data report: liver. Am. J. Transplant. 18(Suppl. 1), 172-253 (2018).

5. Colvin M, Smith JM, Hadley N et al. OPTN/SRTR 2016 annual data report: heart. Am. J. Transplant. 18(Suppl. 1), 291-362 (2018).

6. American Society of Transplantation. Vascularized composite allotransplantation (VCA).

www.myast.org/public-policy/key-position-statements/vascularized-composite-allotransplantation-vca-research

7. Brandacher G. Vascularized composite allotransplantation: a field is maturing. Curr. Opin. Organ Transplant. 23(5), 559-560 (2018). 
8. Valapour M, Lehr CJ, Skeans MA et al. OPTN/SRTR 2016 annual data report: lung. Am. J. Transplant. 18(Suppl. 1), 363-433 (2018).

9. Wey A, Pyke J, Schladt DP et al. Offer acceptance practices and geographic variability in allocation model for end-stage liver disease at transplant. Liver Transpl. 24(4), 478-487 (2018).

10. Organ Procurement and Transplantation Network. National data. https://optn.transplant.hrsa.gov/data/view-data-reports/national-data/

11. Stegall MD, Morris RE, Alloway RR, Mannon RB. Developing new immunosuppression for the next generation of transplant recipients: the path forward. Am. J. Transplant. 16(4), 1094-1101 (2016).

12. Kamila P, Smith SG, Patzer R, Wolf MS, Marina S. Medication regimen complexity in kidney and liver transplant recipients. Transplantation 98(7), e73-e74 (2014).

13. No authors listed. Cyclosporin in cadaveric renal transplantation: one-year follow-up of a multicentre trial. Lancet 2(8357), 986-989 (1983).

14. Kolata G. Drug transforms transplant medicine. Science 221(4605), 40-42 (1983).

15. Hale DA. Basic transplantation immunology. Surg. Clin. North Am. 86(5), 1103-1125 (2006).

16. Lindholm A, Kahan BD. Influence of cyclosporine pharmacokinetics, trough concentrations, and AUC monitoring on outcome after kidney transplantation. Clin. Pharmacol. Ther. 54(2), 205-218 (1993).

17. Uptodate. Pharmacology of cyclosporine and tacrolimus (2019). www.uptodate.com/contents/search

18. Kuypers DR, Naesens M, De Jonge H, Lerut E, Verbeke K, Vanrenterghem Y. Tacrolimus dose requirements and CYP3A5 genotype and the development of calcineurin inhibitor-associated nephrotoxicity in renal allograft recipients. Ther. Drug Monit. 32(4), 394-404 (2010).

19. Kuypers DR, De Jonge H, Naesens M, Vanrenterghem Y. A prospective, open-label, observational clinical cohort study of the association between delayed renal allograft function, tacrolimus exposure, and CYP3A5 genotype in adult recipients. Clin. Ther. 32(12), 2012-2023 (2010).

20. Barrera-Pulido L, Aguilera-Garcia I, Docobo-Perez F et al. Clinical relevance and prevalence of polymorphisms in CYP3A5 and MDRI genes that encode tacrolimus biotransformation enzymes in liver transplant recipients. Transplant. Proc. 40(9), 2949-2951 (2008).

21. Thervet E, Loriot MA, Barbier S et al. Optimization of initial tacrolimus dose using pharmacogenetic testing. Clin. Pharmacol. Ther. 87(6), 721-726 (2010).

22. Pallet N, Etienne I, Buchler M et al. Long-term clinical impact of adaptation of initial tacrolimus dosing to CYP3A5 genotype. Am. J. Transplant. 16(9), 2670-2675 (2016).

23. Shuker N, Bouamar R, Van Schaik RH et al. A randomized controlled trial comparing the efficacy of CYP3A5 genotype-based with body-weight-based tacrolimus dosing after living donor kidney transplantation. Am. J. Transplant. 16(7), 2085-2096 (2016).

24. MeltDose ${ }^{\circledR}:$ US7217431 (2007).

25. Grinyo JM, Petruzzelli S. Once-daily LCP-Tacro MeltDose tacrolimus for the prophylaxis of organ rejection in kidney and liver transplantations. Expert Rev. Clin. Immunol. 10(12), 1567-1579 (2014).

26. Kidney Disease: Improving Global Outcomes (KDIGO) Transplant Work Group. KDIGO clinical practice guideline for the care of kidney transplant recipients. Am. J. Transplant. 9(Suppl. 3), S1-S155 (2009).

27. Budde K, Bunnapradist S, Grinyo JM et al. Novel once-daily extended-release tacrolimus (LCPT) versus twice-daily tacrolimus in de novo kidney transplants: one-year results of Phase III, double-blind, randomized trial. Am. J. Transplant. 14(12), 2796-2806 (2014).

28. Bunnapradist S, Rostaing L, Alloway RR et al. LCPT once-daily extended-release tacrolimus tablets versus twice-daily capsules: a pooled analysis of two Phase 3 trials in important de novo and stable kidney transplant recipient subgroups. Transpl. Int. 29(5), 603-611 (2016).

29. Hale DA. Basic transplantation immunology. Surg. Clin. North Am. 86(5), 1103-1125 (2006).

30. Wagner M, Earley AK, Webster AC, Schmid CH, Balk EM, Uhlig K. Mycophenolic acid versus azathioprine as primary immunosuppression for kidney transplant recipients. Cochrane Database Syst. Rev. CD007746 (2015).

31. Khush KK, Cherikh WS, Chambers DC et al. The International Thoracic Organ Transplant Registry of the International Society for Heart and Lung Transplantation: Thirty-fifth Adult Heart Transplantation Report-2018; focus theme: multiorgan transplantation. J. Heart Lung Transplant. 37(10), 1155-1168 (2018).

32. Leroy C, Rigot JM, Leroy M et al. Immunosuppressive drugs and fertility. Orphanet. J. Rare Dis. 10, 136 (2015).

33. Flannigan KL, Taylor MR, Pereira SK et al. An intact microbiota is required for the gastrointestinal toxicity of the immunosuppressant mycophenolate mofetil. J. Heart Lung Transplant. 37(9), 1047-1059 (2018).

34. Kelly PA, Gruber SA, Behbod F, Kahan BD. Sirolimus, a new, potent immunosuppressive agent. Pharmacotherapy 17(6), 1148-1156 (1997).

35. Flechner SM, Glyda M, Cockfield S et al. The ORION study: comparison of two sirolimus-based regimens versus tacrolimus and mycophenolate mofetil in renal allograft recipients. Am. J. Transplant. 11(8), 1633-1644 (2011).

36. Flechner SM, Gurkan A, Hartmann A et al. A randomized, open-label study of sirolimus versus cyclosporine in primary de novo renal allograft recipients. Transplantation 95(10), 1233-1241 (2013). 
37. Strueber M, Warnecke G, Fuge J et al. Everolimus versus mycophenolate mofetil de novo after lung transplantation: a prospective, randomized, open-label trial. Am. J. Transplant. 16(11), 3171-3180 (2016).

38. Flechner SM. mTOR inhibition and clinical transplantation: kidney. Transplantation 102(2S Suppl. 1), S17-S18 (2018).

39. Uptodate. Pharmacology of mammalian (mechanistic) target of rapamycin (mTOR) inhibitors (2019). www.uptodate.com/contents/search

40. Knoll GA, Kokolo MB, Mallick R et al. Effect of sirolimus on malignancy and survival after kidney transplantation: systematic review and meta-analysis of individual patient data. BMJ 349, g6679 (2014).

41. Qi WX, Huang YJ, Yao Y, Shen Z, Min DL. Incidence and risk of treatment-related mortality with mTOR inhibitors everolimus and temsirolimus in cancer patients: a meta-analysis. PLoS ONE 8(6), e65166 (2013).

42. Badve SV, Pascoe EM, Burke M et al. Mammalian target of rapamycin inhibitors and clinical outcomes in adult kidney transplant recipients. Clin. J. Am. Soc. Nephrol. 11(10), 1845-1855 (2016).

43. Milliman. 2017 US organ and tissue transplant cost estimates and discussion: august 2017. http://us.milliman.com/uploadedFiles/insight/2017/2017-Transplant-Report.pdf

44. Evans RW, Applegate WH, Briscoe DM et al. Cost-related immunosuppressive medication nonadherence among kidney transplant recipients. Clin. J. Am. Soc. Nephrol. 5(12), 2323-2328 (2010).

45. Annema C, Drent G, Roodbol PF et al. Trajectories of anxiety and depression after liver transplantation as related to outcomes during 2-year follow-up: a prospective cohort study. Psychosom. Med. 80(2), 174-183 (2018).

46. Scheel JF, Schieber K, Reber S et al. Psychosocial variables associated with immunosuppressive medication non-adherence after renal transplantation. Front. Psychiatry 9, 23 (2018).

47. Howell M, Wong G, Turner RM et al. The consistency and reporting of quality-of-life outcomes in trials of immunosuppressive agents in kidney transplantation: a systematic review and meta-analysis. Am. J. Kidney Dis. 67(5), 762-774 (2016).

48. Tasciotti E, Cabrera FJ, Evangelopoulos $\mathrm{M}$ et al. The emerging role of nanotechnology in cell and organ transplantation. Transplantation 100(8), 1629-1638 (2016).

49. Fisher JD, Acharya AP, Little SR. Micro and nanoparticle drug delivery systems for preventing allotransplant rejection. Clin. Immunol. 160(1), 24-35 (2015).

50. Patel K, Atkinson C, Broome A, McGillicuddy J, Chavin K, Nadig S. Utilization of machine perfusion and nanotechnology for liver transplantation. Curr. Transpl. Rep. 2(4), 303-311 (2015).

51. Braza M, Lameijer M, Perez-Medina C et al. Rapamycin nanotherapy delays heart transplant rejection [abstract]. Am. J. Transplant. 16(Suppl. 3), (2016).

52. Braza MS, Van Leent MMT, Lameijer M et al. Inhibiting inflammation with myeloid cell-specific nanobiologics promotes organ transplant acceptance. Immunity 49(5), 819.e6-828.e6 (2018).

53. Gajanayake T, Olariu R, Leclere FM et al. A single localized dose of enzyme-responsive hydrogel improves long-term survival of a vascularized composite allograft. Sci. Transl. Med. 6(249), 249ra110 (2014).

54. Feturi FG, Weinstock M, Zhao W et al. Mycophenolic acid for topical immunosuppression in vascularized composite allotransplantation: cptimizing formulation and preliminary evaluation of bioavailability and pharmacokinetics. Front. Surg. 5, 20 (2018).

55. Das NA, Peters JI, Simmons JD et al. The efficacy of inhaled nanoparticle tacrolimus in preventing rejection in an orthotopic rat lung transplant model. J. Thorac. Cardiovasc. Surg. 154(6), 2144.e1-2151.e1 (2017).

56. Hayes D Jr, Zwischenberger JB, Mansour HM. Aerosolized tacrolimus: a case report in a lung transplant recipient. Transplant. Proc. 42(9), 3876-3879 (2010).

57. Bayer J, Das NA, Baisden CE et al. Effect of inhaled tacrolimus on ischemia reperfusion injury in rat lung transplant model. J. Thorac. Cardiovasc. Surg. 146(5), 1213-1219, discussion 1219 (2013).

58. Iacono AT, Johnson BA, Grgurich WF et al. A randomized trial of inhaled cyclosporine in lung-transplant recipients. N. Engl. J. Med. 354(2), 141-150 (2006).

59. Iacono AT, Corcoran TE, Griffith BP et al. Aerosol cyclosporin therapy in lung transplant recipients with bronchiolitis obliterans. Eur. Respir. J. 23(3), 384-390 (2004).

60. Iacono AT, Keenan RJ, Duncan SR et al. Aerosolized cyclosporine in lung recipients with refractory chronic rejection. Am. J. Respir. Crit. Care Med. 153(4 Pt 1), 1451-1455 (1996).

61. Crespo-Leiro MG, Stypmann J, Schulz U et al. Performance of gene-expression profiling test score variability to predict future clinical events in heart transplant recipients. BMC Cardiovasc. Disord. 15, 120 (2015).

62. Deng MC, Elashoff B, Pham MX et al. Utility of gene expression profiling score variability to predict clinical events in heart transplant recipients. Transplantation 97(6), 708-714 (2014).

63. Austin BA, Arnold PJ, Kao A. The impact of time post cardiac transplant on gene expression profile scores, an analysis of 32,043 tests. J. Cardiovasc. Dis. Diagn. 1(3), 1000114 (2013). 
64. Crespo-Leiro M, Zuckermann A, Bara C et al. Concordance among pathologists in the second cardiac allograft rejection gene expression observational study (CARGO II). Transplantation 94(11), 1172-1177 (2012).

65. Costanzo MR, Dipchand A, Starling R et al. The International Society of Heart and Lung Transplantation Guidelines for the care of heart transplant recipients. J. Heart Lung Transplant. 29(8), 914-956 (2010).

66. Grskovic M, Hiller DJ, Eubank LA et al. Validation of a clinical-grade assay to measure donor-derived cell-free DNA in solid organ transplant recipients. J. Mol. Diagn. 18(6), 890-902 (2016).

67. Bloom RD, Bromberg JS, Poggio ED et al. Cell-free DNA and active rejection in kidney allografts. J. Am. Soc. Nephrol. 28(7), 2221-2232 (2017).

68. Bromberg J, Brennan D, Poggio E et al. Biological variation of donor-derived cell-free DNA in renal transplant recipients: clinical implications. J. Applied Lab. Med. 2(2), 1-13 (2017).

69. Tanaka S, Sugimoto $S$, Kurosaki $\mathrm{T}$ et al. Donor-derived cell-free DNA is associated with acute rejection and decreased oxygenation in primary graft dysfunction after living donor-lobar lung transplantation. Sci. Rep. 8, 15366 (2018).

70. Litjens NHR, Huang L, Dedeoglu B, Meijers RWJ, Kwekkeboom J, Betjes MGH. Protective cytomegalovirus (CMV)-specific T-cell immunity is frequent in kidney transplant patients without serum anti-CMV antibodies. Front. Immunol. 8, 1137 (2017).

71. Kim SH, Lee HJ, Kim SM et al. Diagnostic usefulness of cytomegalovirus (CMV)-specific T-cell immunity in predicting CMV infection after kidney transplantation: a pilot proof-of-concept study. Infect. Chemother. 47(2), 105-110 (2015).

72. Chiereghin A, Potena L, Borgese L et al. Monitoring of cytomegalovirus (CMV)-specific cell-mediated immunity in heart transplant recipients: clinical utility of the QuantiFERON-CMV assay for management of posttransplant CMV infection. J. Clin. Microbiol. 56(4), pii: e01040-17 (2018).

73. Brestrich G, Zwinger S, Fischer A et al. Adoptive T-cell therapy of a lung transplanted patient with severe CMV disease and resistance to antiviral therapy. Am. J. Transplant. 9(7), 1679-1684 (2009).

74. Markmann J, Guinan E, Geissler E et al. Initial experience with donor specific tregs in kidney transplantation [abstract]. Am. J. Transplant. 16(Suppl. 3), (2016). https://atcmeetingabstracts.com/abstract/initial-experience-with-donor-specific-tregs-in-kidney-transplantation

75. Rana D, Zreiqat H, Benkirane-Jessel N, Ramakrishna S, Ramalingam M. Development of decellularized scaffolds for stem cell-driven tissue engineering. J. Tissue Eng. Regen. Med. 11(4), 942-965 (2017). 